\title{
Lineamientos estratégicos para las juntas de acción comunal en Barranquilla, Colombia
}

\author{
Sánchez Otero, Madelin*
}

\section{Resumen}

Las Juntas de Acción Comunal (JAC) contribuyen al desarrollo de sus comunidades; sin embargo, en la localidad suroriente, en la ciudad de Barranquilla, Colombia, su accionar se ve afectado por factores políticos y de gestión. Con base en lo anterior, la investigación tuvo como finalidad definir lineamientos estratégicos para la gestión efectiva de las organizaciones en estudio. Para alcanzar el objetivo propuesto, se realizó una investigación de tipo descriptivo, soportada en el método inductivo, con un diseño no experimental de campo. Los hallazgos encontrados revelan que las juntas de acción comunal presentan debilidades, principalmente, en la organización y gestión, lo cual obedece a deficientes competencias de gestión por parte de los miembros, débil estructura organizacional, dificultades financieras y la no efectiva vinculación con la comunidad; además, el Estado ha sido proclive a la promulgación de leyes y decretos para lograr la participación de la sociedad civil, pero al momento de la implementación no se ha logrado la articulación. La conclusión principal del estudio, es que los lineamientos estratégicos son vitales para redimensionar y fortalecer las capacidades de las organizaciones comunitarias en función de la articulación con el Estado y la comunidad que representan para empoderarla de su realidad y puedan gestionar el progreso de su localidad.

Palabras clave: Gestión, estrategia, acción social, juntas de acción comunal, Barranquilla.

\section{Strategic Guidelines for Community Action Boards in Barranquilla, Colombia}

\section{Abstract}

Communal Action Boards Action (JAC) contribute to the development of their communities; however, in the southeast, in the city of Barranquilla, Colombia, their action is affected by political and management factors. This research aimed to define strategic guidelines for effective management of the organizations under study. To achieve this objective, descriptive research was conducted, supported by

\section{Recibido: 15-06-13 Aceptado: 07-05-14}

Administradora de empresas. Especialista en Gestión de Proyectos Educativos. Magister en Dirección Estratégica de la Universidad Internacional Iberoamericana. Docente e investigadora de la Corporación Universidad de la Costa, CUC. Colombia. E-mail: msanchez@cuc.edu.co 
the inductive method, with a non-experimental, field design. Findings reveal that the JAC have weaknesses, mainly in organization and management, due to poor management skills among the members, weak organizational structure, financial difficulties and ineffective community involvement. In addition, the state has tended to promulgate laws and decrees to achieve the participation of civil society, but has not achieved interconnection at the moment of implementation. The main conclusion of the study is that strategic guidelines are vital for resizing and strengthening the capacities of community organizations to coordinate with the State and the community they represent, in order to empower them based on their reality so they can manage the progress of their locality.

Keyword: Management, strategy, social action, communal action boards, Barranquilla.

\section{Introducción}

Durante el mandato de Alberto Lleras Camargo (1958-1962), se crearon las Juntas de Acción Comunal (JAC) en Colombia, por medio de la Ley 19 de 1958. Surgieron en un contexto marcado por el inicio del Frente Nacional y el interés de los gobiernos de promover la participación de la comunidad en las políticas del Estado y lograr disminución de costos de los programas sociales, como prevención de acciones violentas.

La acción participativa se impulsó mucho más en Colombia con la Constitución de1991, en la cual se contempla la participación ciudadana como elemento dinamizador del Estado. Con ella, se da vida a diferentes formas de acción como son: las JAC y los comités de participación comunitaria, entre otros mecanismos. El objetivo principal fue incentivar la democracia con el fin que muchas de las acciones de la comunidad fueran orientadas hacia las necesidades reales, dejando de lado los vicios políticos.

Las JAC se conciben desde dos enfoques: en un primer escenario, son reconocidas como organizaciones comunitarias; por otro lado, como organizaciones del Estado. Según Jaramillo (2009), frente a estas organizaciones prevalecen dos miradas: una propuesta por Borrero (1999) y Leal et al., (1990), la cual se mantiene vigente; y señala que este tipo de organizaciones son espacios privilegiados para el clientelismo y la política tradicional. Más que en organizaciones autónomas, se constituyen en mecanismos a través de los cuales el Estado y los partidos políticos se relacionan con las comunidades y garantizan el intercambio de beneficios por votos.

La otra mirada, según Londoño (1994), es que estas organizaciones responden a la búsqueda de fórmulas colectivas para la satisfacción de necesidades básicas. Estas fórmulas se manifiestan tanto en la solidaridad familiar y vecinal, como en la conformación de organizaciones con grados diferentes de formalidad.

El escenario actual en el que se desarrollan las actividades de las organizaciones sin ánimo de lucro es cambiante y turbulento, sumado a ello, la declaración de los objetivos del milenio de la Organización de las Naciones Unidas, como también el pacto mundial, ha suscitado que el tercer sector se dinamice y tenga mayor interconectividad. A esa realidad, no son ajenas las JAC de la localidad suroriente del Distrito de Barranquilla en el 
Departamento del Atlántico. Situación que sin duda obliga a las organizaciones a ser más efectivas en sus intervenciones.

De igual modo, ser capaces de plasmar, ejecutar y monitorear planes de largo plazo, implica gestionar y establecer estrategias efectivas para lograr resultados superiores. Al respecto, Sandoval et al., (2012:663) afirman: "La idea es que las organizaciones sean capaces de acoplarse a la estructura del modelo de gestión, que se pueda utilizar para adaptar y poner en práctica las acciones necesarias para cumplir con los objetivos estratégicos".

Autores como Chandler (1962), Ansoff (1984), Porter (1996), Druker (1999), y Blejmar (2009), entre otros, han abordado el tema de estrategia y gestión durante décadas. La gestión y la estrategia representan el guion para las acciones de una organización, a su vez, la estrategia permite definir el marco de actuación de quienes son responsables de ejecutarla.

En ese sentido, el objetivo de este estudio es definir lineamientos estratégicos para mejorar la gestión de las juntas de acción comunal, considerando como eje central el impulso del pensamiento estratégico de los líderes comunitarios y su reflexión sobre qué hacer y cómo hacerlo a través de la definición de objetivos, estrategias, proyectos, acciones y recursos.

La metodología utilizada en el desarrollo de la investigación se fundamentó en un diseño no experimental de campo, de tipo descriptivo y proyectivo, el cual tiene elementos de descripción, registro, análisis e interpretación de la natu- raleza actual de la composición o proceso de los fenómenos sobre conclusiones dominantes o cómo una persona, grupo, institución o cosa se conduce o funciona en el presente (Rodríguez, 2005).

A partir del estudio de estos elementos se intenta proponer soluciones a la problemática identificada. En este orden de ideas, fue necesaria la elaboración de instrumentos y estrategias para la recolección de la información como fueron: realización de mesas de trabajo con asociados y miembros, entrevistas a presidentes de las JAC, aplicación de cuestionarios y foros de discusión a la comunidad. Con la finalidad de apoyar lo antes expuesto, se llevó a cabo las siguientes actividades en el marco de la investigación: socialización de la propuesta de investigación ante miembros de JAC, capacitación en gestión social, orientada a generar competencias gerenciales para la promoción de desarrollo local, analizar las condiciones externas e internas en las que operan esta organizaciones, a fin de establecer la situación nacional, regional y local; de igual forma, se identificaron las capacidades internas y se realizó acompañamiento en la formulación de planes estratégicos.

Dado lo anterior, se realizó un diagnóstico de 15 JAC con la participación de la comunidad, sus líderes, el apoyo de la Alcaldía Mayor y la alcaldía de la localidad. Con la información obtenida, su análisis y el apoyo en bases de datos se formularon lineamientos para una mejor gestión de las JAC. La selección de las organizaciones que constituyó la muestra intencional, se hizo teniendo en cuenta los avances, su dinamismo, prácticas di- 
ferentes a las demás y posibilidades de suministro de la información para el objetivo del estudio.

Por otro lado, los resultados obtenidos pueden contribuir a impulsar el diseño de políticas públicas en materia del fortalecimiento a organizaciones de base, concretamente a las juntas de acción comunal.

\section{Juntas de Acción Comunal en Colombia: Concepto, historia y evolución}

En esta sección se abordarán tres temas de las juntas de acción comunal. Inicialmente, se realiza una conceptualización del término, luego se realiza un recorrido histórico especificando los aspectos más relevantes en cada periodo, finalmente se concluye con una descripción de su evolución.

Según la Ley 743 de 2002 emitida por el congreso de la Republica de Colombia (2002), por la cual se desarrolla el Artículo 38 de la Constitución Política de Colombia en lo referente a los organismos de Acción Comunal, las JAC se definen como una organización cívica y comunitaria sin ánimo de lucro, para el ejercicio de la democracia participativa, la cual está integrada por personas de una localidad para buscar el desarrollo integral, sostenible y sustentable de sus comunidades a través del esfuerzo conjunto.

Los inicios de las JAC en Colombia datan desde 1954, en ese período el Comité Nacional de Planeación y el Presidente de la República solicitaron al Centro de Investigación Francés "Economía y Humanismo", elaborar un estudio sobre las necesidades y posibilidades de desarrollo en Colombia (Cardona, 2010). Den- tro de los resultados del estudio en mención, el humanista Lebret, quien lideraba la misión, propuso la creación de las juntas de acción comunal.

La recomendación de Lebret es consolidada entre 1955 y 1958. Al respecto, dos sociólogos de la Universidad Nacional de Colombia, Orlando Fals Borda y Camilo Tórres, impulsaron la creación de las primeras juntas de acción comunal, las que se iniciaron en el Municipio de Chocontá y más tarde en barrios del sur de Bogotá como San Benito, Tunjuelito y Meissen (Cardona, 2010). Estas primeras acciones fueron respaldadas en el Gobierno de Alberto Lleras Camargo, a través de la Ley 19 de 1958 (Congreso de Colombia 1958), que permitió la creación de las Juntas de Acción Comunal en Colombia. En esa época se vivió una fuerte violencia debido a la muerte del candidato a la presidencia Jorge Eliécer Gaitán, y las juntas de acción comunal sirvieron como puente para logar la paz entre los partidos liberales y conservadores.

El desarrollo histórico de las Juntas de Acción Comunal, según el Consejo Nacional de Política Económica y Social de Colombia-Conpes 3661 (2010), Política Nacional para el Fortalecimiento de los organismos de Acción Comunal, se define en cuatro etapas así:

1. Etapa de 1958 a 1970: La autogestión. El inicio de las juntas de acción comunal, surgió de manera espontánea por personas de la sociedad civil, quienes decidieron gestionar y dar solución a problemáticas de las comunidades populares que representaban.

2. Etapa de 1970 a 1991: La clientelización. En esta etapa se disminuyó la autogestión y nació el "clientelismo", 
es decir, las juntas de acción comunal se convirtieron en instrumentos de los políticos para ganar adeptos y favores políticos.

3. Etapa de 1991 a 2002: Fortalecimiento institucional de la participación y declive financiero. Con el cambio que se dio institucionalmente, se dan nuevas espacios para que las personas civiles participen y de igual forma mecanismos para el control social de las acciones de la gestión pública.

4. Etapa de 2002 a 2009. Reconstrucción. En esta etapa nace la Ley 743 de 2002 (Congreso de la República de Colombia, 2002) la cual define el cómo debe operar y el fortalecerse la acción comunal. Se consideran aspectos como la personería jurídica y su sostenibilidad.

En estas últimas décadas, prevalecen dos enfoques de las juntas de acción comunal: el primero, es que infortunadamente muchas instituciones siguen mi- rando a las organizaciones de acción comunal-OAC-como simple instrumento de uso para sus intereses políticos, como actor y sujeto fundamental de la convivencia y el desarrollo; el segundo, hace referencia a las personas que participan en acción comunal con la convicción plena que a través de este mecanismo se puede cambiar la realidad o dar solución a las necesidades de las comunidades, toda vez que se gestione ante el Estado (Cardona, 2005).

A pesar de lo expresado en párrafos anteriores, las juntas de acción comunal han tenido un crecimiento exponencial en Colombia, tal como lo señala el gráfico1: el número de organizaciones que existía en el país para 1965 era de 3.837 , luego en el año 1982 la cifra pasó a 21.554, y para el 2008 se contaban con 53.865. Sin embargo, este crecimiento se ha dado de manera desorganizada en términos de su funcionamiento, aunado a que sus acciones han sido limitadas pues no tienen soportes económicos significativos (Valencia, 2010) (Gráfico 1).

\section{Gráfico 1 \\ Crecimiento de las Juntas de Acción Comunal 1958-2008}

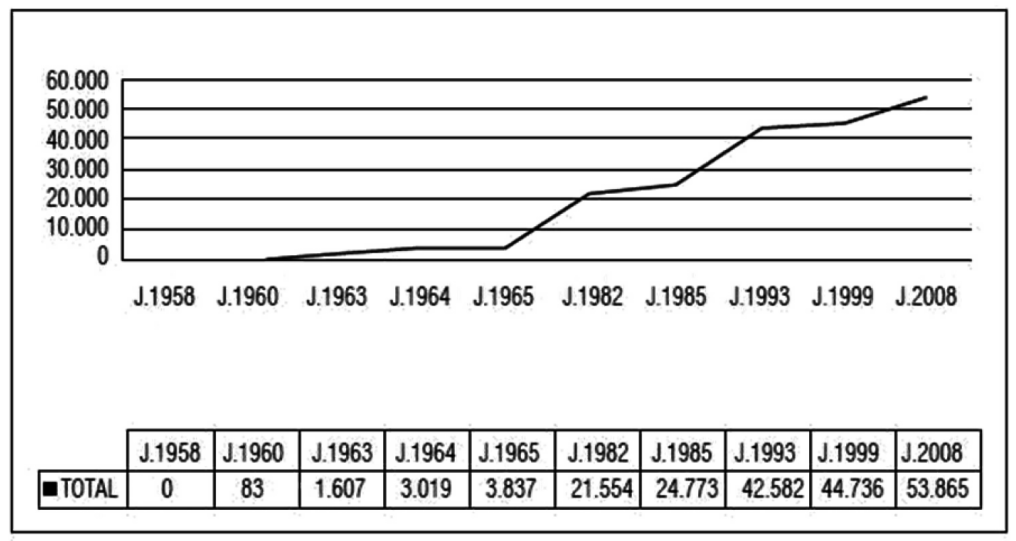

Fuente: Valencia (2010:204). 
Con respecto a los datos estadísticos a nivel de país en cuanto a las JAC, se requiere de cifras puntuales y actualizadas, que realmente caractericen las condiciones en las que operan estas organizaciones y se contrasten con los retos propuestos desde la normatividad vigente que existe en Colombia para el impulso de las mismas y desde luego de la participación ciudadana. Valencia (2010:201) expresa: "La acción comunal tiene futuro, por consiguiente, es útil conocer algunos de los pilares o fundamentos de este horizonte social, puntualizando aspectos relevantes de su propia trayectoria, desarrollo y metas en la dimensión y profundidad de aproximarse a identificar, más que investigar, el proceso sobre la acción comunal de dónde viene, dónde está y para dónde va".

Finalmente, desde las políticas públicas y sus procedimientos, se necesitan acciones concretas para que los organismos de acción comunal actúen como puente de comunicación y mediación con el propósito de mejorar de manera eficaz las condiciones sociales de los ciudadanos. Sobre todo, "aquellos sectores de la población que dejaron de recibir los beneficios de las políticas públicas o alcances de la gestión gubernamental y que fueron, en unos casos insuficiente, en otros ineficiente o ambos a la vez" (Valero, 2012:531).

\section{Aproximación al concepto de gestión y estrategia}

En este punto, se desarrollará el concepto de gestión y estrategia considerando autoridades en el tema. Se resalta la importancia de la articulación de estas dos variables en los resultados de las organizaciones.

\subsection{Gestión}

Para Blejmar (2009:15) la gestión implica las acciones necesarias para lograr resultados. Es decir, quien gestiona hace. Pero su hacer fundamental es hacer para que con los otros se haga del modo más potente posible". De lo anterior se infiere que a través de la gestión se busca la excelencia y eficiencia a través de otros. Sin embargo, Ginebra (2012) plantea que pensar en la excelencia es un error y que hay una realidad que pocos libros de gestión abordan como es la incompetencia. Hamel (2012) considera que es tarea de la gestión que los empleados aprendan y avancen. Por tanto, la gestión exige de quienes dirigen habilidades para ello. Al respeto Fernández (2009) indica que los directivos necesariamente tienen que mirar más allá en su proceso de gestión. Para Ocaña (2012) la gestión es hacer realidad lo que la dirección plantea, realizando las acciones que se tienen que llevar a cabo.

En resumen, la revisión de los diferentes autores permite identificar elementos comunes en sus concepciones de gestión: a) Proyección: implica establecer objetivos o resultados a los que quiere llegar la organización; b) Toma de decisiones: referida a los soluciones que define quien administra para dar respuesta a problemáticas o situaciones concretas o bajo incertidumbre; c) Recursos: necesarios para alcanzar todo lo anterior, y finalmente, el pensamiento estratégico, clave para la gestión.

En general, la gestión implica actuar, es decir, tener claro el objetivo o la meta que se quiere lograr y hacerlo realidad. Al respecto, quien dirige juega un 
papel fundamental en los resultados. Si es capaz de visualizar como quiere que sea su organización y de ejecutarlo, obtendrá resultados, en el caso específico de las JAC en pro de las comunidades que representan.

\subsection{Estrategia}

En el mundo académico y de negocios la estrategia ha sido estudiada de manera amplia. La importancia de la estrategia es el papel fundamental que tiene en las organizaciones, marca las pautas para alcanzar los objetivos. En tal sentido, se hace revisión en la literatura de la conceptualización de varios autores respecto al tema.

Para Ansoff (1984) la estrategia es el punto de unión entre la organización y el entorno, en tanto se identifica los negocios actuales y futuros en los que la organización puede incursionar. Mintzberg et al., (2007:3), afirman que la estrategia es "el patrón de una serie de acciones que ocurren en el tiempo". Esta idea, implica pensar de manera estratégica, es decir, los empresarios o administradores son clave para delinear o establecer en el tiempo estos patrones de manera consciente para lograr llevar a la organización a un nivel superior.

En la revisión de autores se encuentra también, Drucker (1999), quien afirma que con la estrategia se persigue alcanzar los resultados de la organización en un contexto no predecible. Es decir, la estrategia surge porque no se tiene certeza de lo que sucederá, por lo que no hace falta estrategia para algo predeterminado, esto se consigue a través del proceso administrativo.
En cambio, para Porter (2011: 103) "la estrategia consiste en ser diferente. Significa la selección deliberada de un conjunto de actividades distintas para entregar una mezcla de valor". En relación con estas dos últimas definiciones, se analizan también dos aspectos no mencionados antes por los otros autores, y son importantes porque dan fundamento a la estrategia: 1) Un contexto no predecible; 2) Posición única a través de valor.

En síntesis, en el ámbito de la literatura sobre la revisión de la concepción de estrategia de diferentes autores, relacionada con las organizaciones, se evidencian varios aspectos comunes: a) estudiar la realidad, es decir, revisar aspectos internos y externos que afectan a la organización de manera positiva o negativa; b) el pensamiento estratégico asociado a la imaginación y la creatividad, habilidad clave para que los directivos puedan llevar a las organizaciones a niveles superiores; $c$ ) definición de un marco estratégico: misión, visión, objetivos, políticas; d) diseño de planes a largo plazo, lo que contribuye a no improvisar; e) asignación de recursos, fundamental para que se cumpla lo planeado y, f) seguimiento, asegurarse de lograr lo planeado o en caso contrario hacer las acciones correctivas necesarias.

Desde esta perspectiva, la sinergia entre gestión y estrategia es necesaria y obligatoria. Una buena estrategia y la consolidación de la misma están ligadas a la gestión realizada por los miembros de la organización. Es decir, la articulación de la estrategia con la ejecución son claves para el logro de los objetivos de cualquiera organización. En tal sentido, dependiendo del buen manejo de estas 
dos variables, la organización podrá "ser autosostenible" e impactar positivamente a la comunidad beneficiaria.

En esa sinergia el principal responsable de todo este proceso es el individuo, específicamente quien dirige la organización; el pensamiento estratégico ayuda a comprender que lo estratégico y la gestión contribuyen a la organización y hacen parte de un entorno que deben adaptarse de forma rápida para responder de manera adecuada a las demandas de sus grupos de interés.

\section{Diagnóstico y lineamientos estratégicos para las Juntas de Acción Comunal}

Las organizaciones sin ánimo de lucro han logrado tener un papel fundamental en la sociedad, buscando a través de sus acciones reducir la desigualdad y la pobreza, así como el fortalecimiento del Estado democrático y el fortalecimiento de una ciudadanía plena, incluyente y responsable, aspectos que ha vislumbrado el Instituto Interamericano para el Desarrollo Económico y Social (INDES). En tal sentido, Quiñones (2002) manifiesta que muchos países no dan respuestas a las necesidades sociales por falta de recursos, situación que ha incrementado la participación ciudadana a través de organizaciones civiles.

Muchas organizaciones sin ánimo de lucro no logran realmente el propósito por el cual se crean, no cuentan con recursos, y sus dirigentes no tienen las competencias para gestionar la organización y mucho menos para generar los recursos que requieren para la operación y las actividades encaminadas a sus beneficiarios.
Según Pinilla (2011), estas organizaciones trabajan en un entorno tan competitivo que deben hacer grandes esfuerzos por obtener recursos de donaciones, gremios, empresas, entre otros, aspecto que representa debilidad para muchas y se relaciona directamente con el crecimiento $y$ sostenibilidad de las mismas.

Las JAC de Colombia, específicamente las de Barranquilla hacen parte de este contexto. Una de las principales debilidades de las Juntas de Acción Comunal se fundamenta en su organización y gestión. En el Cuadro 1 se hace análisis de los resultados encontrados en la investigación en cuanto a la situación que presentan las JAC en estudio, tanto a nivel macro, meso y micro.

En el nivel macro, el actor principal es el Estado. Los entrevistados manifestaron que existen políticas públicas para la acción comunal en Colombia, pero no se hace efectiva, y el Estado no responde a las necesidades reales que enfrentan las JAC. Por ejemplo: la falta de recursos, la modernización, según ellos el Estado las creó, pero se olvidó de ellas.

En lo que respecta al nivel meso, el actor principal son los entes municipales. Las principales dificultades que afrontan las JAC al respecto son: falta de apoyo a las actividades proyectadas o realizadas, en especial la asignación de recursos, la articulación entre las JAC y los diferentes actores sociales, y otro no menos importante, los intereses políticos.

A nivel micro, se encuentra como actor principal la Juntas de Acción Comunal, de las cuales se ocupa el presente estudio. Las principales dificultades que presentan las organizaciones en este nivel son: insuficiente capacidad de ges- 


\section{Cuadro 1}

\section{Situación de las Juntas de Acción Comunal en Barranquilla, 2011}

\begin{tabular}{|c|c|c|}
\hline Nivel & Actores & Problemática \\
\hline Macro & Estado & $\begin{array}{l}\text { Aplicabilidad de la política pública para organismo de acción Comunal. } \\
\text { Asignación de recursos. } \\
\text { Modernización de las JAC }\end{array}$ \\
\hline Meso & $\begin{array}{l}\text { Entidades } \\
\text { Municipales }\end{array}$ & $\begin{array}{l}\text { Apoyo a las acciones de las JAC } \\
\text { Asignación de los recursos } \\
\text { Descoordinación de las acciones que adelantan los grupos u organi- } \\
\text { zaciones en las comunidades, lo que genera duplicidad de esfuerzos, } \\
\text { poca racionalización de los recursos y atomización de las actividades. } \\
\text { Interés político. }\end{array}$ \\
\hline Micro & $\begin{array}{c}\text { Juntas de acción } \\
\text { Comunal }\end{array}$ & $\begin{array}{l}\text { Capacidad de gestión, vinculación de la comunidad a las actividades } \\
\text { de las JAC. Imagen ante la comunidad, competencias gerenciales de } \\
\text { los miembros, viven el día a día en sus actividades; nulo sistema de } \\
\text { información y no tienen recursos físicos ni monetarios para operar. } \\
\text { Desconocimiento de la sociedad civil respecto a las normas, políticas } \\
\text { y mecanismos de participación para mejorar la situación de sus co- } \\
\text { munidades. }\end{array}$ \\
\hline
\end{tabular}

Fuente: Elaboración propia.

tión, deficiente formación gerencial, escasos recursos, poca participación de la ciudadanía, débil imagen ante la comunidad. Toda esta situación es planteada por la Alcaldía Mayor de Bogotá en estudio realizado (2009:12): "Las estructuras internas de las organizaciones comunales tienden a la dispersión de la base social y a la concentración del poder en los dignatarios, lo que de por sí ya genera problemas de representatividad y contrapesos en el nivel micro de la organización comunal".

El reto con relación a la situación actual de estas organizaciones, es lograr superar estas dificultades a fin que puedan contribuir al desarrollo local de sus comunidades. Al respecto, Villar (2007:5) comenta: "esta participación de organizaciones de base en asuntos públicos tiene en el desarrollo local participativo un es- pacio privilegiado". Esta definición implica una gran tarea para las organizaciones.

Los planteamientos de los autores antes mencionados, contrastan con la realidad que viven muchas comunidades en el país; en el caso que nos ocupa: los barrios de la localidad suroccidente no son la excepción. En este orden de ideas, las organizaciones comunales a través de la gestión y como parte de ella la estrategia, se convierten en elemento transformador de la sociedad, toda vez que se constituyen en una alternativa de solución al problema identificado. Según Navajo (2009) las organizaciones sociales requieren gestionar de manera diferente viejos problemas sociales.

Los sistemas clásicos de gestión son obsoletos para dar respuesta a un entorno hostil y competitivo en el sector 
no lucrativo. Es de destacar que "impulsar el desarrollo local desde la perspectiva cooperativa, implica un reto para las comunidades" (Sánchez, 2010:16). Es un reto porque existen factores como: intereses particulares, poca participación ciudadana, déficit de recursos y factores políticos que inciden de manera directa en acciones de las JAC.

Con base en la importancia que la literatura pertinente otorga a la estrategia y a la gestión, además de considerar el diagnóstico realizado, se formulan los lineamientos estratégicos claves para las JAC (Diagrama 1): a) Fortalecimiento interno de la organización y, b) Consolidación de las relaciones externas; con estos se buscó crear una herramienta que les permitiera direccionar las acciones, a fin de contribuir de manera efectiva al desarrollo de las comunidades locales que representan. Entendiéndose este desarrollo como el conjunto de procesos económicos, políticos, culturales y sociales, que integran los esfuerzos de la población, sus organizaciones y las del Estado, para mejorar la calidad de vida de las co- munidades (Ley 743 de 2002) (Congreso de la República de Colombia, 2002). Estas organizaciones, aunque son sin ánimo de lucro, se diferencian de otras porque no se maneja el concepto de beneficiario; las JAC son organizaciones que buscan impulsar la participación ciudadana en pro del desarrollo local.

De esta manera, es de destacar el fortalecimiento que necesita la organización JAC en todos los aspectos, para poder dar respuestas oportunas y pertinentes a la colectividad que representa. En este orden de ideas, se establecen las acciones concebidas para cada lineamiento:

\subsection{Fortalecimiento interno de la organización}

Orientado a desarrollar capacidades en las organizaciones de base, con la intención de generar desarrollo local a través de la efectiva gestión de las mismas. Revisando el concepto de capacidad organizacional, autores como Horton et al., (2008), afirman que es el la habilidad de una organización para lograr re-

\section{Diagrama 1 \\ Lineamientos estratégicos para Juntas de Acción Comunal en Barranquilla}

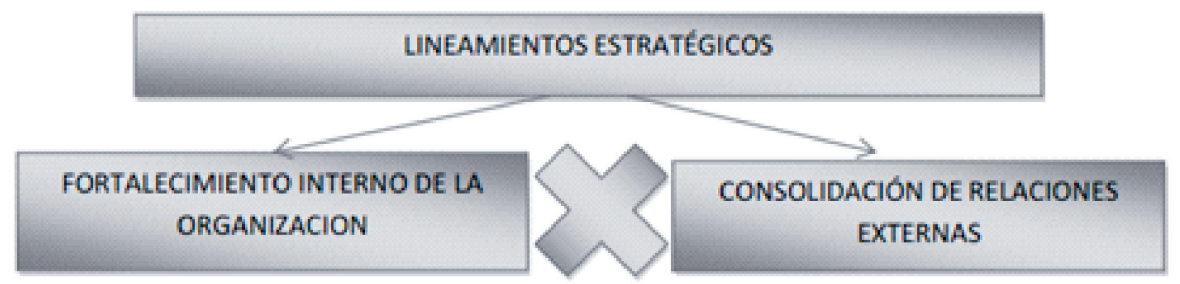

RESULTADOS ESPERADOS

Fuente: Elaboración propia. 
sultados de manera relevante y sostenible. Para Leinwand et al., (2010), es la coherencia de la empresa en función de alinear sus capacidades distintivas, sus recursos, el portafolio de servicios o productos y el mercado.

A partir de la revisión del concepto de capacidad organizacional, se identifican aspectos claves como: habilidad, eficiencia, recursos, fortalezas, debilidades. Por tanto, el rol de los individuos o directivos en este proceso se convierte en eje central, dependiendo de las habilidades de pensamiento estratégico y de la aplicación de este, así será el desarrollo de la organización. El pensamiento estratégico permite anticiparse a los hechos o ver los problemas o situaciones que otros no ven; de igual forma, adaptarse o recuperarse a las situaciones críticas que se presenten. En tal sentido, para este trabajo los referentes teóricos son orientadores para proponer a las JAC desde el ámbito de la capacidad organizacional, acciones en pro de mejorar el desempeño interno y del impacto en lo externo.

Con respecto a lo anterior, para el lineamiento estratégico del fortalecimiento interno de la organización, se definen las siguientes capacidades claves:

1) Proyección de la organización: Las organizaciones que desean ser perdurables y sostenibles en el tiempo crean un imaginario del ideal de organización, y este ideal se cumple en la medida que se defina de manera formal, y se realicen las acciones pertinentes para alcanzarlo. El logro de los resultados está asociado a las habilidades de pensamiento estratégico y a la gestión de quienes dirigen la organización.

Por las características propias de las JAC, que han sido conformadas por los habitantes de una comunidad, debería primar los intereses colectivos y no los particulares, por lo que la proyección de la JAC debe ser de largo plazo y no empezar de cero cada vez que se elige a un nuevo presidente y miembros. Con miras a fortalecer a la organización en estudio, respecto a la proyección, se requiere de las siguientes fases:

1. Definir el rumbo estratégico, teniendo como referencia el marco normativo y las condiciones de la comunidad. En este aparte, se define la visión, misión, valores, objetivos y estrategias, que debe abordarse desde lo participativo, es decir, incluir a la comunidad para mayor objetividad de lo que se desea y de igual forma teniendo claro la posibilidades de ejecución.

2. Análisis de la organización, el cual se divide en interno y externo. En el interno se hace un análisis desde lo organizativo, financiero, infraestructura, personal, comunicación, tecnología; y en la parte externa se analizan y evalúan variables relacionadas con la comunidad, el barrio, como también aspectos sociales, demográficos, económicos, ambientales.

3. Definición de los planes tácticos y operativos; el insumo para el desarrollo de esta fase son los puntos anteriores. A través de ello, se materializa ese sueño. Para ser más específicos, se definen las áreas claves, las metas, acciones, indicadores, responsables y resultados.

4. El seguimiento, para verificar el cumplimiento del plan. Este se puede hacer de manera permanente, el tamaño de la organización lo permite. 
En síntesis, se busca que las acciones identificadas para proyectar la empresa queden por escrito, a través de un documento llamado Plan Estratégico o Plan de Desarrollo de la organización, cuya finalidad, es no improvisar, sino realizar acciones que generen realmente valor a esta organización a largo plazo.

2) Personas: las personas tienen el talento y lo colocan al servicio de las organizaciones, sean con o sin ánimo de lucro, por un beneficio económico o simplemente como voluntario. Entendiendo talento como "aquellas ideas, conocimientos y habilidades que proporcionan a la persona el potencial para producir un valor desproporcionado con los recursos que sus organizaciones le facilitan" (Goffee et al., 2007:72).

En lo que se refiere a las JAC, toda persona vinculada a estas no tiene remuneración, sino que trabaja por mejorar las condiciones de su comunidad. En particular, las personas vinculadas a estas organizaciones y el resto de la comunidad necesitan trabajar en equipo. Para los primeros, es importante el desarrollo de habilidades de liderazgo y gestión social; para los segundos, conocer los instrumentos de participación ciudadana que existen en su país, máxime, cuando el entorno exige de competencias a los individuos para empoderarse de su realidad y buscar las soluciones a ella. Por ejemplo, aspectos claves de gobernabilidad, equidad, transparencia, cooperación, solidaridad, entre otros, son fundamentales para el ejercicio de la democracia participativa.

Por todo lo expresado, las personas de la comunidad tienen un rol trascedente para la consolidación de estas organizaciones en sus respectivas comuni- dades y los miembros activos o dirigentes actuales deben vincular a la comunidad en sus procesos y desarrollar procesos de comunicación de manera efectiva.

Como consecuencia, el desarrollo de competencias en liderazgo y gestión social para los miembros de las JAC y los que aspiren a ella, es una necesidad urgente. El desarrollo de competencias para la gestión es clave, porque permite tomar mejores decisiones estratégicas y así mismo dirigir con éxito las competencias de los otros.

Es decir, el presidente y los miembros de las JAC deberían tener competencias para la gestión de personas y desde luego para llevar a la organización a un nivel superior; esto implica tener habilidades como visión y pensamiento estratégico para identificar, proponer y ejecutar acciones en pro del desarrollo local. Estas competencias son "esenciales para la supervivencia de la organización en un mundo siempre cambiante y más competitivo" (Montecinos, 2012:350).

Lo anterior indica la importancia de las competencias de liderazgo y gestión social de los presidentes o miembros de la JAC para impulsar equipos auto-gestionados en la comunidad, con la finalidad ejecutar el plan estratégico. El desafío, entonces es, vincular de manera efectiva a la comunidad para conformar comités por áreas estratégicas del plan y así poder optimizar recursos y fortalecer los procesos internos y externos de la organización.

En síntesis, la implementación de todos estos factores debe estar orientada hacia el trabajo en equipo, la competitividad, la autogestión, el desarrollo, el crecimiento y la sostenibilidad. Por tanto, la definición de equipos auto-gestionados fue la fórmula sugerida para operar; es 
decir, el grupo de personas que conforman las JAC representa un área estratégica, logrando así involucrar a la comunidad en cada equipo conformado.

3) Programas y proyectos: tiene como objetivo identificar, diseñar, proponer, ejecutar y supervisar programas y proyectos necesarios para la comunidad, articulando con instituciones u organizaciones públicas o privadas que realicen o pueden realizar acciones en la comunidad, así mismo realizar vigilancia tecnológica de convocatorias de cofinanciación de proyectos para contribuir al desarrollo sostenible de la comunidad.

Vale la pena hacer hincapié en lo expresado en el ítem de personas, en el caso particular de los programas y proyectos, identificar qué personas de la comunidad tienen experiencia en elaboración, implementación, evaluación de proyectos y programas locales, nacionales e internacionales, que tengan la capacidad de coordinar y liderar redes de trabajo. Ahora bien, si este talento no se encuentra en la comunidad, los líderes deben ubicar este talento en las universidades, instituciones del Estado u otras organizaciones que tengan programas hacia al fortalecimiento de la comunidad en temas de proyectos; por ejemplo: alcaldía distrital y local, fundaciones, cooperativas y Servicio Nacional de Aprendizaje (SENA).

\subsection{Consolidación de las relaciones externas}

Este lineamiento propende a generar o consolidar las relaciones, que de hecho debe establecer la junta para ofrecer mejores posibilidades a la comunidad que representa. Por ello, es determinante la identificación de diferentes actores clave. Esta identificación debe realizarse basado en el propósito, los programas y proyectos a desarrollar por la junta; es decir, deben estar enmarcados en las acciones del lineamiento anterior. Será preciso entonces, realizar análisis de quiénes son los actores que tendrán rol importante en la consecución del propósito de la junta. Una vez hecho este análisis se debe generar una propuesta de valor que resulte atractiva para ese actor teniendo en cuenta su naturaleza.

El propósito de ello, es la búsqueda de cooperación y mediación. A continuación se detallan las acciones a emprender por las JAC con actores clave:

1. Comunidad local: constituida por participantes y facilitadores de los procesos de desarrollo local, por lo cual se requiere vincularlos de manera efectiva en las acciones de la JAC. Para eso es necesario establecer: a) Puentes claros de comunicación como la utilización de folletos o boletines con información relevante y entregarla en cada vivienda; perifoneo, página web, mantenerse en redes de sociales, organizar eventos de integración por lo menos cada dos meses, en el cual se integra la comunidad y se pueden mostrar avances de las actividades desarrolladas o futuras de la JAC, y ubicar en los sitios públicos del barrio cartelera informativa; b) Impulsar capacitaciones sobre los mecanismos de participación que tiene el ciudadano; c) Impulsar campañas para mejorar la seguridad de sus barrios.

2. Empresas: Identificar y vincular las empresas de la zona mediante los programas de responsabilidad social 
que tienen estas; para ello, la JAC debe formular o tener en ejecución un proyecto atractivo. La empresa se comprometerá a dar recursos o a participar, sólo si ve la seriedad de un buen proyecto y si hay aliados estratégicos reconocidos.

3. Instituciones del Estado: La JAC debe propender por la cooperación del Estado y la comunidad para mejorar las condiciones del barrio; de igual forma la corresponsabilidad de los actores en el desarrollo local. Para este propósito, involucrar de manera efectiva a la alcaldía distrital y local es tarea prioritaria del presidente o miembros de las JAC. Otra entidad con la que por necesidad tienen que hacer alianzas es el Servicio Nacional de Aprendizaje- SENA, pues esta entidad formativa ofrece un portafolio de capacitaciones en aspectos técnicos que si la comunidad en conjunto con la JAC tienen identificado cuáles son las necesidades de capacitación en los jóvenes y mujeres jefas de hogar, podrán aprovechar para mejorar las competencias de la población mencionada y desarrollar proyectos productivos.

4. Universidades: A través de sus unidades de proyección social realizan actividades para fortalecer las comunidades, y de igual forma aportan estudiantes en práctica que podrían fortalecer los procesos internos y eternos de las JAC. Además, tienen el talento del que adolecen las JAC.

5. Organizaciones sin ánimo de lucro: han aumentado de manera significativa en los últimos años; dedican tiempo, acciones y recursos para contri- buir la reducción de la pobreza; por ello, la JAC debe trabajar de manera mancomunada con estas organizaciones que tengan presencia en la comunidad. A estas organizaciones les interesa trabajar en red, porque está en la "búsqueda de oportunidades para mejorar la calidad de vida y las condiciones" (Sánchez, 2011) de los menos favorecidos.

6. Colegios: Identificar y realizar alianzas estratégicas con los colegios de la zona, a fin de promover la participación de jóvenes y docentes en la solución de problemas de su comunidad y se apropien de los mecanismos de participación ciudadana, mediante la realización de mesas de trabajo, talleres, seminarios, actividades lúdicas y jornadas pedagógicas; todo esto tiene como propósito ir vinculado a los jóvenes para el relevo generacional de líderes comunitarios; hasta el momento, en las JAC el mayor porcentaje de participación ciudadana es de adultos mayores.

7. JAC vecinas: Mantener contacto permanente o realizar alianzas con JAC vecinas, para la búsqueda de soluciones conjuntas a problema comunes; además, compartir experiencias de las prácticas de gestión.

En definitiva, el proceso entre las JAC-entorno se convierte en herramienta fundamental para el logro del propósito de las organizaciones, porque vincula efectivamente los actores claves en resolución de problemáticas en la comunidad. El resultado esperado de las acciones que corresponden al lineamiento equivalente a la consolidación de las relaciones externas son: Promover la participación 
ciudadana, la cooperación interinstitucional y el desarrollo de proyectos para la mejora de las condiciones de la comunidad en todos los aspectos, contribuyendo así a optimizar los recursos de muchos mediante la coordinación de actividades.

Para lograr sinergia de los lineamientos internos y externos en una estructura organizacional simple por la naturaleza y los pocos recursos que tienen estas organizaciones, a fin de que respalde la ejecución de las acciones, políticas concretas, procedimientos, manuales, medios y técnicas que se deben implementar.

En síntesis, los lineamientos planteados se fundamentan en un ciclo de gestión integral que contempla aspectos internos y externos, administrativos, de organización proyección y metas, enfocados desde la comunidad, el desarrollo, la sostenibilidad y la gestión. La adopción de los lineamientos estratégicos por parte de las organizaciones en estudio debe dar como resultado final mejorar el desempeño de la organización e interactuar de manera efectiva en la comunidad. Todo eso se traduce en desarrollar una mejor operación de los procesos internos, contribuir y generar una adecuada relación con su entorno para lograr así generar ventajas mutuas.

Otro aspecto clave para alcanzar o consolidar los resultados que se pretenden, es el concurso de las instituciones del Estado que tienen relación directa con las JAC. Estas tienen que repensar la forma en la que interactúa con las comunidades. Es decir, valorar como afirma Montecinos (2012:351) el "aprendizaje de cómo infundir una nueva manera de pensar a la gerencia pública para crear autoaprendizaje, y una organización con capacidad de adaptación y forjadora de conocimiento", que finalmente se traduzca en beneficios reales para los ciudadanos y no quede solo en la política.

Finalmente, todo proceso debe ser evaluado a fin de cambiar o reestructurar la estrategia; por ello, entre los lineamientos se contemplan como parte importante del proceso la retroalimentación, seguimiento y control, entendiéndose que los cambios dados en el contexto son constantes e invitan a la definición de propuestas flexibles. Los lineamientos son orientación o guías que contribuyen al pensamiento y a la toma de decisiones, que debe ser medido, pero no son camisa de fuerza. Los resultados esperados o deseados apuntan a buscar la eficacia y eficiencia de las acciones que realicen estos organismos de base. En ese sentido, todos los lineamientos deben conducir a lograr transformación o impacto en las comunidades a través de la buena gestión integral que hagan los miembros de las JAC.

\section{Conclusiones}

La investigación realizada ha permitido identificar que la organización y gestión son factores de principal debilidad en las Juntas de Acción Comunal; esto se debe a que no tienen una sostenibilidad financiera, deficiencias en su estructura, deficientes competencias en gestión por parte de los miembros, no hay efectiva vinculación de los ciudadanos; además, el Estado no ha podido responder de forma adecuada a sus propuestas intersectoriales, situación que no conlleva efectivamente al desarrollo local de las 
comunidades en las que se encuentran inmersas.

A partir de este resultado, puede entenderse que existe una brecha entre la situación real de las JAC y la concepción y mandato de la Ley: las Juntas de Acción Comunal se crean con el direccionamiento de promover la participación ciudadana en las políticas del Estado y de generar desarrollo.

En este contexto, los principales resultados del estudio indican que la Junta de Acción Comunal es tan solo uno de los muchos escenarios para el ejercicio del poder. Allí no se toman decisiones que afecten a grandes poblaciones, tampoco se manejan vastos capitales. Sin embargo, representan para muchas veredas del país, el espacio donde tienen lugar las primeras prácticas políticas, el escenario para la construcción y el ejercicio del poder y posibilitan dimensionar lo que ocurre en estructuras cuya incidencia está mucho más allá del vecindario.

En igual sentido, los hallazgos obtenidos evidencian que las JAC manifiestan necesidades de las comunidades que demandan acciones concretas, por tal razón, deben potencializarse las capacidades de gestión que se hace necesario tengan dentro de su comunidad y en su interrelación con el aparato estatal. En este sentido, debe existir un empoderamiento de la sociedad civil en la construcción del tejido social que contribuya al progreso de sus capacidades de desarrollo y de gestión.

De este estudio se han derivado importantes contribuciones al campo de la gestión y de la estrategia de las JAC. Es decir, los lineamientos estratégicos planteados para las juntas de acción comunal contribuyen con el desarrollo de acciones claras desde lo interno y externo, orienta- das al desarrollo local sostenible y la articulación de las JAC con el sector público, el sector educativo, las organizaciones y la empresa privada, buscando así la consolidación de la organización.

Se sugiere que las acciones comunales sean más proactivas y participativas en su gestión, a fin de lograr una transformación en las comunidades y afianzar sus capacidades para determinar los objetivos que persiguen y las estrategias que utilizarán para alcanzarlos.

Con el propósito de lograr sinergia, las relaciones entre Estado y sociedad civil necesariamente requieren de un cambio para lograr mayor cooperación. El Estado debe ser el veedor de las prácticas políticas, así mismo, innovar en estrategias de desarrollo para lograr dar respuesta a la demandas de los ciudadanos; y la comunidad debe desarrollar capacidades para participar activamente en la solución de sus propios problemas y en las decisiones de política pública. En relación a lo anterior, los resultados obtenidos podrían orientar a los diferentes actores en el proceso de acción comunal, a fin de lograr que las organizaciones de base cumplan su papel en la sociedad.

En cuanto a futuras líneas de investigación, se propone hacer análisis comparativos entre ciudades de Colombia con otros países, respecto a cómo operan las organizaciones de base y su impacto en las comunidades. También, cómo desde la innovación en la gestión pueden ser autosostenbiles en todos sus aspectos. Por otro lado, es importante estudiar diferentes factores o aspectos que tienen incidencia en la consolidación de las JAC. En tal sentido, se deben estudiar variables como: participación ciudadana, 
comportamientos prosociales, culturales, financieros, rol de las instituciones del Estado, entre otros.

\section{Referencias bibliográficas}

Alcaldía Mayor de Bogotá (2009). ¿Cómo participan las Juntas de Acción Comunal de Santa Fé? Línea base de participación local. Serie: ¿Cómo participan las Juntas de Acción Comunal en Bogotá? Instituto Distrital de la Participación y Acción Comunal (IDPAC).

Ansoff, Igor (1984). Implanting Strategic Management. New Jersey. Prentice Hall International.

Blejmar, Bernardo (2009). Gestionar es hacer que las cosas sucedan: competencias, actitudes y dispositivos para diseñar instituciones. Buenos Aires. Centro de publicaciones Educativas y Material didáctico.

Borrero, Camilo (1999). Acción Comunal y política estatal: ¿Un matrimonio indisoluble? Bogotá, Colombia. Centro de Investigación y Educación Popular-CINEP.

Cardona, Moreno (2010). La Acción Comunal vista desde los congresos comunales. Un sueño auténtico de derechos y democracia. Bogotá, Colombia. Instituto Distrital de la Participación y Acción Comunal IDPAC.

Cardona Moreno (2005). El futuro de las Organizaciones Comunales. Fundador del Movimiento Comunal y Comunitario de Colombia. Ponencia presentada en la jornada pedagógica comunal. Villeta 1, 2, 3 de mayo 2005.

Congreso de la Republica de Colombia (2002). Ley 743 del 5 de junio del 2002, por la cual se desarrolla el Artículo 38 de la Constitución Política de Colombia en lo referente a los organismos de Acción Comunal. Bogotá, Colombia.
Congreso de Colombia (1958). Ley 19 de noviembre de 1958 Sobre reforma administrativa. Bogotá, Colombia.

Consejo Nacional de Política Económica y Social (2010). Conpes 3661. Política Nacional para el fortalecimiento de los organismos de acción comunal. Colombia.

Chandler, Alfred (1962). Strategy and Structure. Chapters in the History of the American Industrial Enterprise. Cambridge, Mass. The Mit Press.

Drucker, Peter (1999). Los desafíos de la Administración en el siglo XXI. Buenos Aires. Editorial. Sudamericana.

Fernández, Juan (2009). Gestión en tiempos de crisis (o cómo dar le la vuelta a la tortilla). Cuadernos gestión del conocimiento.

Ginebra, Gabriel (2012). El japonés que estrelló el tren para ganar tiempo. Barcelona. Editorial Conecta.

Goffee, Rob y Jones, Garret (2007). Leading clever people. Harvard Business Review, 85(3), pp. 72-79.

Hamel, Gary (2012). Lo que importa ahora: Cómo triunfar en un mundo de cambios implacables, competencia feroz e innovación sin barreras, Bogotá. Editorial Norma.

Horton, Douglas; Alexaki, Anastasia; BenettLartey, Samuel; Noele, Kim; Campilan, Dindo ; Carden, Fred ; de Souza, Jose; Thanh, Le ; Khadar, Ibrahim ; Maestry, Albina ; Kayes, Imrul ; Pérez. Jocelyn; Somarriba, Matilde; Vernooy, Ronnie ; Watts, Jamie (2008). Evaluación del desarrollo de capacidades. Experiencias de organizaciones de investigación y desarrollo alrededor del mundo. Cali, Colombia Centro internacional de Agricultura.

Jaramillo, Olga (2009). El ejercicio del poder en las juntas de acción comunal rurales: el caso del Municipio de Sonsón, Antioquia. Bogotá, Colombia. Pontificia Universidad Javeriana, Fa- 
cultad de Estudios Ambientales y Rurales, Maestría en desarrollo rural.

Leal, Francisco y Dávila, Andrés (1990). Clientelismo. El sistema político y su expresión regional. Bogotá: Tercer Mundo. Instituto de Estudios Políticos y Relaciones Internacionales. Colombia. Universidad Nacional.

Leinwand, Cesar (2010). The Essential Advantage: How to Win with a Capabilities-Driven Strategy.

Londoño, Rocío (1994). Una visión de las organizaciones populares en Colombia. Escuela de Liderazgo Democrático. Bogotá, Colombia. Editorial Gazeta.

Mintzberg, Henry; Quinn, James; y Voyeur, John (2007). El proceso estratégico: conceptos, contextos y casos. México, Prentice-Hall Hispanoamericana, p.3.

Montecinos, Egon (2012). Gestión municipal participativa: Entre la nueva gerencia pública y la gobernanza. Revista Venezolana de Gerencia (RVG). Año 17 (58), Universidad del Zulia. PP. 345-364.

Navajo, Pablo (2009). Planificación estratégica en organizaciones no lucrativas: Guía participativa basada en valores. Editorial Narcea Madrid.

Ocaña, Jose (2012). Gestión de proyectos con mapas mentales. Volumen I. Alicante. Editorial el Club Universitario.

Pinilla, Maricela (2011). Contribución del marketing social a la sostenibilidad de fundaciones sin ánimo de lucro: un estudio de caso. Tesis presentada como requisito parcial para optar al título de Magister en Administración en la Universidad Nacional de Colombia-Sede Manizales.

Porter, Michael (2011). ¿Qué es la estrategia? Harvard Business Review. Boston, Massachusetts. pp. 100-117.

Quiñone, Lisandro (2002). Organización de la Sociedad Civil, mecanismos e instru- mentos sobre filantropía y control social: legislación. Instituto tecnológico de Santo Domingo-INTEC.

Rodríguez, Ernesto (2005). Metodología de la investigación: la creatividad, el rigor del estudio y la integridad son factores que transforman al estudiante en un profesionista de éxito. México. Universidad Juárez Autónoma de Tabasco.

Sánchez, Madelin (2010). Situación actual y perspectivas de la cooperativas micro. Revista Económicas CUC. Editorial Educosta.

Sánchez, Madelin (2011). Gestión estratégica en la Fundación Social Amishaday. Revista Económicas CUC. Editorial Educosta.

Sandoval, Franklin; Montaño, Nora; Miguel, Vanessa y Ramos, Esmeralda. (2012). Gestión de perfiles de cargos laborales basados en competencias. Revista Venezolana de Gerencia (RVG). Año 17, № 60, Universidad del Zulia, p. 660-675.

Valencia, Luis (2010). Hacia la modernización orgánica y el fortalecimiento democrático de la Acción Comunal en Colombia. Administración \& Desarrollo. Apuntes Institucionales. Bogotá, Colombia. Escuela Superior de la Administración Púbica, ESAP. 38(52) pp. 201-210.

Valero, Johan. (2012). Programa Barrió Adentro I: una estrategia de gestión pública en el Estado Zulia. Revista Venezolana de Gerencia (RVG). Universidad del Zulia. Año 17. № 59, pp. 529547.

Villar, Rodrigo (2007). Vinculando el desarrollo de base con el desarrollo local: estrategias de intervención de los miembros de Rede América. Fundación para el Desarrollo Institucional de Organizaciones Sociales (Fundación DIS). 